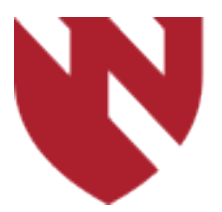

December 2019

\title{
Was It the Beer or the Burgers?
}

Ian Cormier

University of Nebraska Medical Center

Craig Baumgart

University of Nebraska Medical Center

Michael Smith

University of Nebraska Medical Center

Tell us how you used this information in this short survey.

Follow this and additional works at: https://digitalcommons.unmc.edu/gmerj

Part of the Higher Education Commons, and the Medicine and Health Sciences Commons

\section{Recommended Citation}

Cormier, I., Baumgart, C., , Smith, M. Was It the Beer or the Burgers?. Graduate Medical Education Research Journal. 2019 Dec 13; 1(1).

https://digitalcommons.unmc.edu/gmerj/vol1/iss1/56

This Conference Proceeding is brought to you for free and open access by DigitalCommons@UNMC. It has been accepted for inclusion in Graduate Medical Education Research Journal by an authorized editor of DigitalCommons@UNMC.For more information, please contact digitalcommons@unmc.edu. 


\section{Was It the Beer or the Burgers? \\ Creative Commons License \\ (c) (i)@(ङ)}

This work is licensed under a Creative Commons Attribution-Noncommercial-No Derivative Works 4.0 License. 
an established sensitivity of $80.9 \%$ and specificity of $57.1 \%$ in differentiating high-grade gliomas from tumefactive demyelinating lesions. Some studies suggest that even specimen biopsies may be inconclusive, as there are many histopathologic similarities between tumefactive demyelinating lesions and high-grade gliomas. Diffusion tensor imaging (DTI), though not yet utilized in common practice, can be highly sensitive in differentiating these lesions.
Conclusions: For both patient scenarios, MR imaging was insufficient to make a definitive diagnosis, resulting in a delay in treatment. The cases and literature review demonstrate the diagnostic challenges of these conditions using standard MRI alone, and raise awareness of DTI and other non-invasive modalities that may become integrated into future clinical practice.

https://doi.org/10.32873/unmc.dc.gmerj.1.1.053

\section{Utilization of Brain Imaging in Evaluating Patients with Psychogenic Nonepileptic Spells Danmeng Wei, Matthew Garlinghouse, Wenyang Li, Nicholas Swingle, Kaeli K. Samson, Olga Taraschenko}

Mentor: Olga Taraschenko

Program: Neurology

Background: Psychogenic non-epileptic spells (PNES) are paroxysmal movements or sensory events that resemble epileptic seizures but lack corresponding ictal electrographic changes. Patients are often referred for brain imaging tests which contribute to the high cost of care.

Methodology: This is a retrospective chart review of 225 adult patients diagnosed with PNES without epileptic seizures from 2012 to 2017. The frequency of the brain imaging tests prior to vEEG was assessed across all semiological classes and was correlated with other clinical characteristics.

Result: The most prevalent PNES events were characterized by semi-rhythmic small amplitude movements in the extremities (32\%) followed by those resembling clonictonic seizures $(27.7 \%)$. Patients with sensory changes had more imaging tests than those with primitive gesturing and axial posturing. Patients with 3 or more psychiatric disorders had more combined MRIs and CTs prior to diagnosis than patients with two or fewer psychiatric co-morbidities $(\mathrm{p}=0.03)$.

Conclusion: The frequency of brain imaging obtained prior to the definitive diagnosis of PNES is influenced by semiology and the psychiatric health of patients. The PNES with minimal paroxysmal movements in the settings of multiple psychiatric co-morbidities represent particularly challenging patient phenotype which is linked to excessive referrals for brain imaging.

https://doi.org/10.32873/unmc.dc.gmerj.1.1.054

\section{Disseminated Strongyloides Causing Diffuse Alveolar Hemorrhage Mollie Brittan, Brian Boer, Deepak Chandra}

\section{Mentor: Brian Boer}

Program: Internal Medicine

Case presentation: An elderly patient with a past medical history of leukocytoclasic vasculitis on high dose steroids presented with altered mental status. The patient was found to be in septic shock and was admitted to an intensive care unit. The patient was started on broad spectrum antibiotics but continued to decline and developed hemoptysis along with hypoxic respiratory failure requiring intubation. Bronchoscopy revealed frank bronchial and alveolar hemorrhage. Bronchoscopy fluid was sent for microscopy and it revealed nematodes consistent with Strongyloides. The patient was started on

\section{Was It the Beer or the Burgers? Ian Cormier, Craig Baumgart, Michael Smith}

\section{Mentor: Michael Smith \\ Program: Internal Medicine}

Acute pancreatitis is a commonly encountered diagnosis and is the most common gastrointestinal reason for hospitalization. Approximately $75 \%$ of cases of acute pancreatitis are caused by gallstones oral Ivermectin and Albendazole but failed to improve on this regimen and was switched to subcutaneous Ivermectin after FDA approval. The patient ultimately improved and was discharged.

Discussion: Strongyloides stercolis is a nematode that can cause disease in humans. Strongyloides infection can involve cutaneous, pulmonary and gastrointestinal systems. This organism has a unique lifecycle where the parasite enters the host often through the skin and migrates to both the gastrointestinal and pulmonary systems at various points during its lifecycle. This unique life cycle also involves gastrointestinal autoinfection. Autoinfection occurs when a human is infected with a helminth (i.e.
Strongyloides) and the whole parasite's lifecycle takes place within the human host. This process is exaggerated in humans when cell mediated immunity is impaired with immunosuppression (in the case of our patient with corticosteroids). It has the ability to spread to multiple organs during the hyperinfection stage which can cause multisystem organ failure.

Conclusion: Diffuse alveolar hemorrhage caused by disseminated Strongylioides has a high mortality rate and treatment with subcutaneous Ivermectin contributed to her survival as the oral formulation had poor absorption due to gastrointestinal involvement of the parasite.

https://doi.org/10.32873/unmc.dc.gmerj.1.1.055 or alcohol consumption. In contrast, hypertriglyceridemia only accounts for $2-4 \%$ of acute pancreatitis cases.

A middle-aged patient with a history of recurrent acute pancreatitis secondary to alcohol abuse was admitted for acute epigastric abdominal pain consistent with his prior episodes of pancreatitis. Workup was significant for multiple electrolyte derangements and laboratory workers commented on the patient's serum appearing lactescent. Repeat point-of-care testing revealed normal electrolyte levels. A lipid panel revealed profound hypertriglyceridemia and the patient was treated with an insulin 
infusion and fibrate prior to discharge. Elevated triglycerides should always be considered when determining the etiology of acute pancreatitis, even in patients who consume alcohol as excessive alcohol consumption can lead to a state of hypertriglyceridemia. This case also illustrates the interesting relationship between elevated serum lipids and measured serum electrolytes. The presence of elevated lipids increases the non-aqueous components of the serum with a concomitant decrease in the fraction of serum composed of free water and electrolytes. This phenomenon, termed the "electrolyte exclusion effect", is common with the use of flame photometry or indirect potentiometry, the methods utilized by many hospital laboratories. However, this artifact does not occur with the use of direct potentiometry, as this method directly measures the concentration of electrolytes in the water phase of serum. Point-of-caretesting is more accurate in the setting of severe hypertriglyceridemia as it utilizes direct ion-selective electrodes. This can be used in the setting of hypertriglyceridemia or hyperproteinemia to obtain more precise results.

https://doi.org/10.32873/unmc.dc.gmerj.1.1.056

\section{A Cough May be Just the Tip of the Iceberg}

Ian Cormier, Neil Bhogal, Michael Smith

Mentor: Michael Smith

Program: Internal Medicine

Pyogenic liver abscesses (PLAs) can be diagnostically challenging, as they may present with non-specific symptoms and are less frequently encountered than other types of infections. PLAs caused by atypical organisms may be rare and can be associated with a variety of other underlying pathologies including occult malignancy.

A middle-aged patient presented with two weeks of productive cough, dyspnea, subjective fevers and chills. The patient was started on antibiotics for community acquired pneumonia but decompensated with signs of sepsis. Computed tomography (CT) of the chest, abdomen and pelvis revealed multiple bilateral pulmonary nodules with early cavitation concerning for septic emboli and a large multiloculated hepatic hypodensity in the right lobe of the liver concerning for a liver abscess. Anaerobic cultures from an aspirate of the lesion grew Fusobacterium nucleatum. The patient was discharged on intravenous antibiotics along with an outpatient colonoscopy to evaluate for occult malignancy. PLAs, which are typically polymicrobial, are the most common type of liver abscess in the U.S. Many PLAs result from portal vein pyemia, usually related to bowel leakage and peritonitis. Biliary infection and hematogenous spread are other common etiologies. Fusobacterium spp. are anaerobic gram negative rods that occur as normal oral flora and have been associated with periodontal infections. However, they are uncommon causes of pyogenic liver abscesses. PLAs have a known association with GI malignancies and Fusobacterium nucleatum has recently been shown to promote colorectal carcinogenesis by affecting genes associated with the adenoma-carcinoma sequence. The oral cavity could be a potential source of infection for this patient. However, occult GI malignancy must be ruled out, as there is an association between PLAs and malignancy of the gastrointestinal tract. https://doi.org/10.32873/unmc.dc.gmerj.1.1.057

\section{Efficacy of Endoscopic Ultrasonography Guided Gastroenterostomy in Gastric Outlet Obstruction Dhaliwal Amaninder, Cormier lan, Jhand Aravdeep, Rangray Rajani, Bhat Ishfaq, Girotra Mohit, Singh Shailender}

Mentor: Amaninder Dhaliwal

Program: Internal Medicine

Introduction: Gastric outlet obstruction (GOO) is the complication of benign and malignant diseases. Laproscopic gastrojejunostomy and enteral stenting has been the standard of care. Endoscopic ultrasonography guided gastroenterostomy (EUS-GE) has emerged as a newer technique in patients with GOO. Thus, we conducted systematic review and meta-analysis to report the overall technical success, clinical success and adverse events.

Methods: Multiple electronic databases (MEDLINE, EMBASE and Google
Scholar) and conference abstracts were comprehensively searched to identify studies reporting EUS-GE in patients with GOO. References from identified studies were then manually searched for any additional studies. Primary outcome of our meta-analysis was to see the overall pooled technical and clinical success of the procedure. Secondary outcome was to look at the total adverse events of the procedure. The meta-analysis was performed using Der Simonian and Laird random effect model.

Results: 10 studies were included in the final meta-analysis reporting a total of 263 patients (males 135 and females 128) with median age of 61.2 years. The median follow up was 110 days. Overall pooled technical and clinical success rates of the procedure were $92.4 \%$ $(95 \% \mathrm{CI}=89-95, \mathrm{I} 2=0)$ and $88.5 \%(95 \% \mathrm{CI}=$ $84.5-92.5, \mathrm{I} 2=0)$. The total adverse events were $8.1 \%(95 \% \mathrm{CI}=4.1-11.9, \mathrm{I} 2=23 \%)$ mainly as stent migration $(\mathrm{n}=2)$ and peritoneal stent deployment $(\mathrm{n}=15)$.

Conclusion: EUS-GE is a safe and effective technique in patients with GOO with overall high technical and clinical success rates (92.4\%, 88.5\% respectively). However, further randomized control trials are needed to validate these findings.

https://doi.org/10.32873/unmc.dc.gmerj.1.1.058

\section{Environmental Exposures and Respiratory Health}

\section{Letisha J Ferris, Corrine K Hanson; Joan Lappe; Nancy Waltman; Laura Bilek; Jill A Poole}

Mentors: Jill A. Poole and Corrine K. Hanson

Program: Internal Medicine

Background and Objectives: Chronic respiratory diseases lead to co-morbid conditions, including osteoporosis, and environmental exposures are risk factors for respiratory disease development and severity. We evaluated the relationship between environmental exposures and respiratory health in postmenopausal women to better understand how this relationship might impact postmenopausal health outcomes.

\begin{abstract}
Methods: A cross-sectional, sub-study of participants of the Heartland Osteoporosis Prevention Study was conducted whereby questionnaires that included information on agriculture exposures and respiratory health symptoms and diagnoses were mailed and
\end{abstract}

